\title{
Cross sections and single spin asymmetries in hadronic collisions with intrinsic transverse momentum effects
}

\author{
U. D'Alesio and F. Murgia \\ Istituto Nazionale di Fisica Nucleare, Sezione di Cagliari \\ and Dipartimento di Fisica, Università di Cagliari \\ C.P. 170, I-09042 Monserrato (CA), Italy
}

\begin{abstract}
The role of intrinsic transverse momentum both in unpolarized and polarized processes is discussed. We consider inclusive cross sections for pion production in hadronic collisions and for Drell-Yan processes; the results are compared with available experimental data in several different kinematical situations. We reanalyze transverse single spin asymmetries (SSA) observed in inclusive pion production, $p^{\uparrow} p \rightarrow \pi X$, in terms of Sivers effect and show how it can be disentangled in polarized Drell-Yan processes by suitably integrating over some final configurations. Estimates for RHIC experiments are given.
\end{abstract}

In the last years a lot of experimental and theoretical activity has been devoted to the study of transverse single spin asymmetries (SSA) in hadronic collisions and in semi-inclusive DIS. In fact, perturbative QCD (pQCD) with ordinary collinear partonic kinematics leads to negligible values for these asymmetries, as soon as the relevant scale of the process under consideration becomes large. There are however several experimental results which seem to contradict this expectation. Among them let us mention: $i$ ) the large polarization of $\Lambda$ 's and other hyperons produced in $\left.p N \rightarrow \Lambda^{\uparrow} X ; i i\right)$ the large asymmetry observed in $p^{\uparrow} p \rightarrow \pi X$ and $\bar{p}^{\uparrow} p \rightarrow \pi X$; iii) the similar azimuthal asymmetry observed in $\ell p^{\uparrow} \rightarrow \ell \pi X$.

A possible way out from this situation comes from extending the collinear pQCD formalism with the inclusion of spin and partonic intrinsic transverse momentum, $\mathbf{k}_{\perp}$, effects. This leads to the introduction of new spin and $\mathbf{k}_{\perp}$ dependent partonic distribution (PDF) and fragmentation (FF) functions, describing fundamental properties of hadron structure [1].

The role of $\mathbf{k}_{\perp}$ effects in inclusive hadronic reactions has been extensively studied also in the calculation of unpolarized cross sections. It has been shown

* Talk delivered by F. Murgia at the "IX Convegno su Problemi di Fisica Nucleare Teorica", October 9-12, 2002, Cortona, Italy. 
that, particularly at moderately large $p_{T}$ (which is the region where SSA are measured to be large) these effects can be relevant and may help in improving the agreement between experimental results and pQCD (at LO and NLO) calculations, which often underestimate the data [2].

Based on these considerations, in this contribution we present a preliminary account of an ongoing program which aims to describe consistently both polarized and unpolarized cross sections (and SSA) for inclusive particle production in hadronic collisions at large energies and moderately large $p_{T}$, using LO pQCD with the inclusion of intrinsic transverse momentum effects. Our main goal is not to fit the cross sections but rather to show that in our LO approach they are reproduced up to an overall factor of 2-3, compatible with expected NLO K-factors and scale dependences, which reasonably cancel out in SSA and are then out of our present interest.

In a LO pQCD approach at leading twist with inclusion of $\mathbf{k}_{\perp}$ effects, the unpolarized cross section for the inclusive process $A B \rightarrow C X$ reads

$$
\begin{aligned}
d \sigma \propto & \sum_{a, b, c, d} \hat{f}_{a / A}\left(x_{a}, \mathbf{k}_{\perp a}\right) \otimes \hat{f}_{b / B}\left(x_{b}, \mathbf{k}_{\perp b}\right) \\
& \otimes d \hat{\sigma}^{a b \rightarrow c d}\left(x_{a}, x_{b}, \mathbf{k}_{\perp a}, \mathbf{k}_{\perp b}\right) \otimes \hat{D}_{C / c}\left(z, \mathbf{k}_{\perp C}\right)
\end{aligned}
$$

with obvious notations. A similar expression holds for the numerator of a transverse SSA $\left(\propto d \Delta^{N} \sigma / d \sigma\right)$, substituting for the polarized particle involved the corresponding unpolarized PDF (or FF) with the appropriate polarized one, $\Delta^{N} f$ or $\Delta^{N} D$. At leading twist there are four new spin and $\mathbf{k}_{\perp}$ dependent functions to take into account:

$$
\begin{aligned}
\Delta^{N} f_{q / p^{\uparrow}}\left(x, \mathbf{k}_{\perp}\right) & \equiv \hat{f}_{q / p^{\uparrow}}\left(x, \mathbf{k}_{\perp}\right)-\hat{f}_{q / p^{\downarrow}}\left(x, \mathbf{k}_{\perp}\right), \\
\Delta^{N} f_{q^{\uparrow} / p}\left(x, \mathbf{k}_{\perp}\right) & \equiv \hat{f}_{q^{\uparrow} / p}\left(x, \mathbf{k}_{\perp}\right)-\hat{f}_{q \downarrow / p}\left(x, \mathbf{k}_{\perp}\right), \\
\Delta^{N} D_{h / q^{\uparrow}}\left(z, \mathbf{k}_{\perp}\right) & \equiv \hat{D}_{h / q^{\uparrow}}\left(z, \mathbf{k}_{\perp}\right)-\hat{D}_{h / q^{\downarrow}}\left(z, \mathbf{k}_{\perp}\right), \\
\Delta^{N} D_{h^{\uparrow} / q}\left(z, \mathbf{k}_{\perp}\right) & \equiv \hat{D}_{h^{\uparrow} / q}\left(z, \mathbf{k}_{\perp}\right)-\hat{D}_{h \downarrow / q}\left(z, \mathbf{k}_{\perp}\right) .
\end{aligned}
$$

The FF in Eq. (丰) is the Collins function [3], while the PDF in Eq. (2) was first introduced by Sivers [4]. The function in Eq. (3) was considered by Boer [5] and the one in Eq. (5) is the so-called "polarizing" FF [6].

Despite its successful phenomenology, the Sivers function was always a matter of discussions and its very existence rather controversial; in fact in Ref. [3] a proof of its vanishing was given, based on time-reversal invariance (notice that this does not apply to the FF sector). Ways out based on initial state interactions or non standard time-reversal properties [7] were discussed. Very recently a series of papers [8] have resurrected Sivers asymmetry in its full rights.

The natural process to test the Sivers asymmetry is Drell-Yan where there cannot be any effect in fragmentation processes and, by suitably integrating 
over some final configurations, other possible effects vanish. SSA in Drell-Yan processes are particularly important now, as ongoing or imminent experiments at RHIC will be able to measure them.

Let us start considering the role played by the intrinsic $\mathbf{k}_{\perp}$ in the unpolarized cross sections. The PDF and FF in Eq. (1) are given in a simple factorized form, and the $\mathbf{k}_{\perp}$ dependent part is usually taken to have a Gaussian shape:

$$
\hat{f}_{a / A}\left(x, \mathbf{k}_{\perp a}\right)=f_{a / A}(x) \frac{\beta^{2}}{\pi} e^{-\beta^{2} k_{\perp a}^{2}} ; \hat{D}_{q}^{h}\left(z, \mathbf{k}_{\perp h}\right)=D_{q}^{h}(z) \frac{\beta^{\prime 2}}{\pi} e^{-\beta^{\prime 2} k_{\perp h}^{2}}
$$

where the parameter $\beta\left(\beta^{\prime}\right)$ is related to the average partonic (hadronic) $k_{\perp}$ by the simple relation $1 / \beta\left(\beta^{\prime}\right)=\left\langle k_{\perp a(h)}^{2}\right\rangle^{1 / 2}$. Similar expressions are adopted for the polarized PDF and FF of Eq.s (2)-(5).

We have considered several hadronic processes, analyzing a large sample of available data in different kinematical situations. Here we limit ourselves to present few indicative results and comments regarding: i) The Drell-Yan process $p p \rightarrow \ell^{+} \ell^{-} X$; ii) Inclusive pion production in $p p \rightarrow \pi X$.

We find that an overall good reproduction of the corresponding unpolarized cross sections is possible (within the limits indicated above) by choosing, depending on the kinematical situation considered, $\beta=1.0-1.25(\mathrm{GeV} / c)^{-1}$ (that is, $\left\langle k_{\perp}^{2}\right\rangle^{1 / 2}=0.8-1.0 \mathrm{GeV} / c$ ). The choice of $\beta$ is related to the set of PDF utilized; throughout this paper we use the GRV94 set [9]. The optimal choice of $\beta^{\prime}$ in case $i$ ) (pion production) is commented below.

i) At LO and within collinear partonic configuration the final lepton pair produced in Drell-Yan processes cannot have any transverse momentum, $q_{T}$, with respect to the colliding beams. Experimental data show however that the lepton pair has a well defined $q_{T}$ spectrum. As an example, in Fig. 1a we show estimates of the invariant cross section at $E=400 \mathrm{GeV}$ as a function of $q_{T}$, for several different invariant mass bins (in $\mathrm{GeV}$ ) at fixed rapidity $y=0.03$, and using $\beta=1.11(\mathrm{GeV} / c)^{-1}$; data are from [11]. Theoretical curves are arbitrarily raised by a factor $K_{\mathrm{fac}}=1.6$, which could be well accommodated by NLO $\mathrm{K}$-factors and scale dependences, an issue that as said above we do not address here. Notice how data are well reproduced by a Gaussian dependence up to $q_{T}=2-2.5 \mathrm{GeV} / c$; larger $q_{T}$ data show a power-law decrease well explained by pQCD corrections.

ii) For inclusive pion production, $p p \rightarrow \pi X$, some experimental results for SSA are also available, and we can see how our approach works for SSA and unpolarized cross sections at the same time. This case is however more intricate, since we can have $\mathbf{k}_{\perp}$ effects in the fragmentation process also. The $z$ and $\mathbf{k}_{\perp}$ dependences in the FF are chosen according to Eq. (6); a direct $z$ dependence of the $\beta^{\prime}$ parameter seems to be favored, $1 / \beta^{\prime}(z)=\left\langle k_{\perp \pi}^{2}(z)\right\rangle^{1 / 2}=1.4 z^{1.3}(1-z)^{0.2}$ $\mathrm{GeV} / c$.

\footnotetext{
${ }^{\dagger}$ A full account of this analysis, including prompt photon production in $p p \rightarrow \gamma X$, will be presented elsewhere [10].
} 

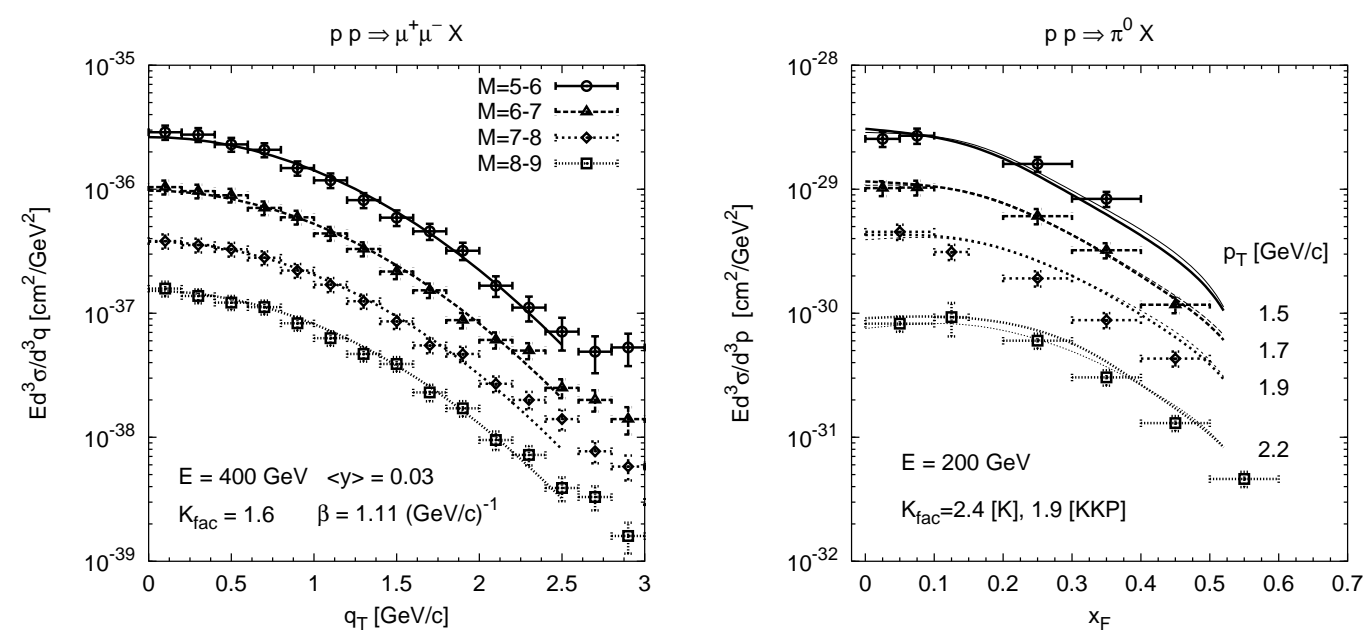

Figure 1: The invariant cross section for (a) $p p \rightarrow \mu^{+} \mu^{-} X$ vs. $q_{T}$ and (b) $p p \rightarrow$ $\pi^{0} X$ vs. $x_{F}$; see plots and text for more details.

Unpolarized FF are presently known with much less accuracy than nucleon PDF. In particular, all available sets of parameterizations for the pion $\mathrm{FF}$ are for neutral pions (or for the sum of charged pions), since $e^{+} e^{-}$data do not allow to separate among $\pi^{+}$and $\pi^{-}$; this can be made under further assumptions, which remain to be tested. In Fig. $1 \mathrm{~b}$ we present estimates of the invariant cross section for the process $p p \rightarrow \pi^{0} X$ at $E=200 \mathrm{GeV}$ vs. $x_{F}$ for different $p_{T}$ values. We use two sets of FF from Kretzer (K, thin lines) [12] and Kniehl, Kramer, and Pötter (KKP, thick lines) [13], $K_{\mathrm{fac}}=2.4(\mathrm{~K}), 1.9(\mathrm{KKP}), \beta=1.25$ $(\mathrm{GeV} / c)^{-1}$. Data are from 14.

Let us now consider the SSA in $p^{\uparrow} p \rightarrow \pi X$, within the same approach and assuming it is generated by the Sivers effect alone, that is from a spin and $\mathbf{k}_{\perp}$ effect in the PDF inside the initial polarized proton, described by the Sivers function $\Delta^{N} f_{q / p^{\uparrow}}\left(x, \mathbf{k}_{\perp}\right)$. Other possible sources for SSA, like the so-called Collins effect, concerning the fragmentation of a polarized parton into the final observed pion, are not considered here. Analogous studies have already been performed [15], using an effective averaging on $\mathbf{k}_{\perp}$ and a simplified partonic kinematics. Here we show the first results with full treatment of $\mathbf{k}_{\perp}$ effects and partonic kinematics. These results are in good qualitative agreement with previous work.

The numerator of the SSA, $d \sigma^{\uparrow}-d \sigma^{\downarrow}$ can be expressed in the form of Eq. (11), with the substitution $\hat{f}_{a / A}\left(x, \mathbf{k}_{\perp}\right) \rightarrow \Delta^{N} f_{q / p^{\uparrow}}\left(x, \mathbf{k}_{\perp}\right)$. For the Sivers function we choose an expression similar to that of the unpolarized distribution, Eq. (6)

$$
\Delta^{N} f_{q / p^{\uparrow}}\left(x, \mathbf{k}_{\perp}\right)=\Delta^{N} f_{q / p^{\uparrow}}(x) h\left(k_{\perp}\right) \sin \phi_{k_{\perp}},
$$

where $\phi_{k_{\perp}}$ is the angle between $\mathbf{k}_{\perp}$ and the polarization vector of the pro- 
ton; $\Delta^{N} f_{q / p^{\uparrow}}(x)$ and $h\left(k_{\perp}\right)$ are such to fulfill the general positivity bound $\left|\Delta^{N} f_{q / p^{\uparrow}}\left(x, k_{\perp}\right)\right| / 2 \hat{f}_{q / p}\left(x, k_{\perp}\right) \leq 1$ :

$$
\begin{gathered}
\Delta^{N} f_{q / p^{\uparrow}}(x)=N_{q} x^{a_{q}}(1-x)^{b_{q}} \frac{\left(a_{q}+b_{q}\right)^{\left(a_{q}+b_{q}\right)}}{a_{q}^{a_{q}} b_{q}^{b_{q}}} 2 f_{q / p}(x), \quad\left|N_{q}\right| \leq 1 \\
h\left(k_{\perp}\right)=\left(2 e \frac{1-r}{r}\right)^{1 / 2} \frac{\beta^{3}}{\pi} k_{\perp} \exp \left[-\beta^{2} k_{\perp}^{2} / r\right], \quad 0<r<1 .
\end{gathered}
$$

A choice of the parameters in Eq.s (8),(9) which allow to reasonably reproduce the experimental results for the pion SSA is the following (only valence quark contributions to the Sivers function are considered):

$$
\begin{aligned}
& N_{u}=+0.5 \quad a_{u}=2.0 \quad b_{u}=0.3 \\
& N_{d}=-1.0 \quad a_{d}=1.5 \quad b_{d}=0.2, \quad r \simeq 0.7 \text {. }
\end{aligned}
$$

In Fig. 2a we show our preliminary estimates of $A_{N}$ with Sivers effect at $E$ $=200 \mathrm{GeV}$ and $p_{T}=1.5 \mathrm{GeV} / \mathrm{c}$, vs. $x_{F}$, for three different choices of the pion FF: K, KKP and a modified version of K. Data are from [16]. The SSA for $\pi^{+}$ and $\pi^{0}$ is well reproduced independently of the FF set. Interestingly, the $\pi^{-}$ case shows a stronger sensitivity to the relation between the leading and nonleading contributions to the fragmentation process, which cannot be extracted from present experimental information. In fact, our results with the $\mathrm{K}(\mathrm{KKP})$ FF sets underestimate (overestimate) in magnitude the $\pi^{-}$asymmetry, while a good agreement is recovered using a somehow fictitious set (K-mod) with an intermediate behavior.

A more direct way to extract the Sivers asymmetry is the analysis of SSA in Drell-Yan processes, that is the production of $\ell^{+} \ell^{-}$pairs in the collision of two hadrons $A$ and $B$ [17]. By considering differential cross sections in the squared invariant mass $\left(M^{2}=\left(p_{a}+p_{b}\right)^{2}\right)$, rapidity $(y)$ and transverse momentum of the lepton pair $\left(\mathbf{q}_{T}\right)$ and integrating out the di-lepton angular dependence, the difference between $d \sigma^{\uparrow}$ for $A^{\uparrow} B \rightarrow \ell^{+} \ell^{-} X$ and $d \sigma^{\downarrow}$ for $A^{\downarrow} B \rightarrow \ell^{+} \ell^{-} X$ from the Sivers asymmetry of Eq. (2), is

$$
\begin{aligned}
& d \sigma^{\uparrow}-d \sigma^{\downarrow}= \\
& \sum_{a b} \int\left[d x_{a} d^{2} \mathbf{k}_{\perp a} d x_{b} d^{2} \mathbf{k}_{\perp b}\right] \Delta^{N} f_{a / A^{\uparrow}}\left(x_{a}, \mathbf{k}_{\perp a}\right) \hat{f}_{b / B}\left(x_{b}, \mathbf{k}_{\perp b}\right) d \hat{\sigma}^{a b \rightarrow \ell^{+} \ell^{-}} .
\end{aligned}
$$

We take the hadron $A$ as moving along the positive $z$-axis, in the $A$ - $B$ c.m. frame and the transverse polarization of hadron $A, \mathbf{P}_{A}$, along the $y$-axis.

In the kinematical regions such that: $q_{T}^{2} \ll M^{2} \ll M_{Z}^{2}$ and $k_{\perp a, b}^{2} \simeq q_{T}^{2}$, the asymmetry becomes

$$
A_{N}=\frac{\sum_{q} e_{q}^{2} \int d^{2} \mathbf{k}_{\perp q} d^{2} \mathbf{k}_{\perp \bar{q}} \delta_{k_{\perp} q_{T}}^{2} \Delta^{N} f_{q / A^{\uparrow}}\left(x_{q}, \mathbf{k}_{\perp q}\right) \hat{f}_{\bar{q} / B}\left(x_{\bar{q}}, \mathbf{k}_{\perp \bar{q}}\right)}{2 \sum_{q} e_{q}^{2} \int d^{2} \mathbf{k}_{\perp q} d^{2} \mathbf{k}_{\perp \bar{q}} \delta_{k_{\perp} q_{T}}^{2} \hat{f}_{q / A}\left(x_{q}, \mathbf{k}_{\perp q}\right) \hat{f}_{\bar{q} / B}\left(x_{\bar{q}}, \mathbf{k}_{\perp \bar{q}}\right)}
$$



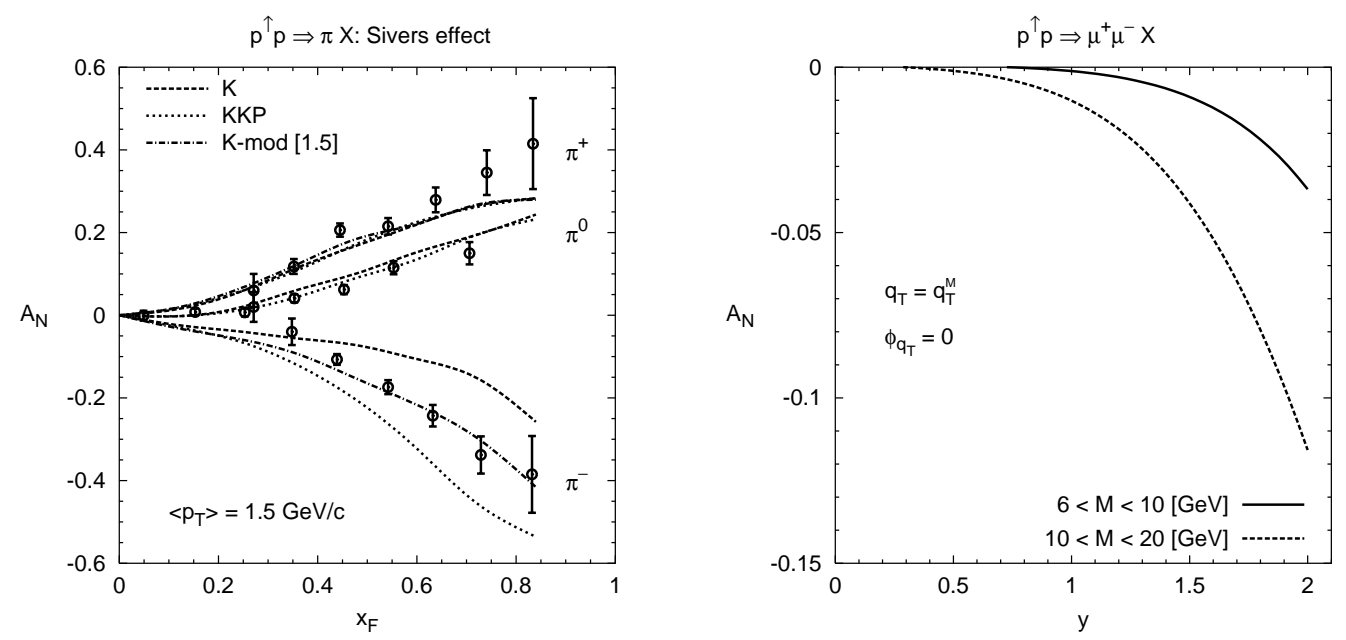

Figure 2: The SSA for (a) $p^{\uparrow} p \rightarrow \pi X$ vs. $x_{F}$ and (b) $p^{\uparrow} p \rightarrow \mu^{+} \mu^{-} X$ vs. $y$; see plots and text for more details.

where $\delta_{k_{\perp} q_{T}}^{2} \equiv \delta^{2}\left(\mathbf{k}_{\perp q}+\mathbf{k}_{\perp \bar{q}}-\mathbf{q}_{T}\right), x_{q} \simeq \frac{M}{\sqrt{s}} e^{y}$ and $x_{\bar{q}} \simeq \frac{M}{\sqrt{s}} e^{-y}$, with $a, b=q, \bar{q}$ and $q=u, \bar{u}, d, \bar{d}, s, \bar{s}$.

Notice that other sources of SSA, like i.e. the distribution function in Eq. (3), would lead to a contribution to $A_{N}$ that vanishes upon integration over all final angular configurations of the $\ell^{+} \ell^{-}$pair.

Inserting the above choice of $\Delta^{N} f\left(x, \mathbf{k}_{\perp}\right)$ and $\hat{f}\left(x, \mathbf{k}_{\perp}\right)$ into Eq. (12) one can perform analytical integrations; being $\beta$ independent of $x$ one gets

$$
\begin{aligned}
A_{N}\left(M, y, \mathbf{q}_{T}\right) & =\mathcal{Q}\left(q_{T}, \phi_{q_{T}}\right) \mathcal{A}(M, y) \\
& =\frac{2 r \sqrt{2 e r(1-r)}}{(1+r)^{2}} \beta q_{T} \cos \phi_{q_{T}} \exp \left[-\frac{1}{2} \frac{1-r}{1+r} \beta^{2} q_{T}^{2}\right] \\
& \times \frac{1}{2} \frac{\sum_{q} e_{q}^{2} \Delta^{N} f_{q / p^{\uparrow}}\left(x_{q}\right) f_{\bar{q} / p}\left(x_{\bar{q}}\right)}{\sum_{q} e_{q}^{2} f_{q / p}\left(x_{q}\right) f_{\bar{q} / p}\left(x_{\bar{q}}\right)}
\end{aligned}
$$

where $\phi_{q_{T}}$ is the azimuthal angle of $\mathbf{q}_{T} \cdot \mathcal{Q}\left(q_{T}\right)$ has a maximum at $q_{T}=q_{T}^{M}=$ $\sqrt{(1+r) /(1-r)} / \beta$, where its value is $\mathcal{Q}\left(q_{T}^{M}\right) \equiv \mathcal{Q}_{M}=[2 r /(1+r)]^{3 / 2}$. Notice that only the position of the maximum depends on $\beta$.

One further uncertainty concerns the sign of the asymmetry: as noticed by Collins and verified by Brodsky [8], the Sivers asymmetry has opposite signs in Drell-Yan and SIDIS, respectively related to $s$-channel and $t$-channel elementary reactions. As in $p-p$ interactions we expect that large $x_{F}$ regions are dominated by $t$-channel quark processes, we think that the Sivers function extracted from $p-p$ data should be opposite to that contributing to D-Y processes. Our numerical estimates will then be given with the same parameters 
as in Eq. (10), changing the signs of $N_{u}$ and $N_{d}$. Given these considerations, even a simple comparison of the sign of our estimates with data might be significant.

In Fig. 2b we show $A_{N}$ at $\sqrt{s}=200 \mathrm{GeV}$ as a function of $y$ averaged over two kinematical ranges $6 \leq M \leq 10 \mathrm{GeV}$ and $10 \leq M \leq 20 \mathrm{GeV}$. We have fixed $q_{T}=q_{T}^{M}(\simeq 1.9 \mathrm{GeV} / c)$, and $\phi_{q_{T}}=0$, which maximizes the $\mathbf{q}_{T}$-dependent part of the asymmetry; on the other hand $A_{N}$ is reduced by a factor of $50 \%$ at $q_{T} \simeq 0.6 \mathrm{GeV} / c$.

We can also consider the asymmetry averaged over $\mathbf{q}_{T}$ up to a value of $q_{T}=q_{T 1}$ (integrating over $\phi_{q_{T}}$ in the range $[0, \pi / 2]$ only, otherwise one would get zero). In our simple model for $q_{T 1} \geq 1.7 \mathrm{GeV} / c$ we would get $\left\langle A_{N}\right\rangle \simeq$ $0.4 A_{N}\left(q_{T}^{M}\right)\left(\right.$ for $\left.q_{T 1}=0.6 \mathrm{GeV} / c\left\langle A_{N}\right\rangle \simeq 0.2 A_{N}\left(q_{T}^{M}\right)\right)$.

Our estimates show that $A_{N}$ can be well measurable within RHIC expected statistical accuracy; the actual values depending on the assumed functional form of the Sivers function and its role with valence quarks.

In conclusion, we have presented here preliminary results of a study of partonic transverse momentum effects both in unpolarized and polarized cross sections (and SSA) for inclusive particle production in hadronic collisions. These results show that it seems possible to reproduce reasonably well, within pQCD at LO and leading twist and up to a factor of 2-3, unpolarized cross sections for Drell-Yan processes, and inclusive pion production in hadronic collisions, in several different kinematical situations. Within the same approach, we have reanalyzed the SSA for $p^{\uparrow} p \rightarrow \pi X$ taking into account Sivers effect alone and found reasonable agreement with data and with previous (simplified) theoretical results that are therefore confirmed by our analysis. We have also shown how SSA in Drell-Yan processes can be a powerful tool to learn on the Sivers asymmetry.

The next steps of this program are the study of the pion SSA with Collins effect, of the SSA in photon production, and of the unpolarized cross section and the transverse polarization for $\Lambda$ production in unpolarized hadronic collisions. The extension of our analysis to RHIC kinematics, where a thorough program on SSA measurements is in progress, is of great interest. First estimates of the SSA in $p^{\uparrow} p \rightarrow \pi X$ seem to be in reasonable agreement with preliminary results from RHIC [18].

\section{Acknowledgments}

This contribution is based on work done in collaboration with M. Anselmino. Support from COFIN. MURST-PRIN is acknowledged. 


\section{References}

[1] For review papers on the subject, see, e.g., Z.-T. Liang and C. Boros, Int. J. Mod. Phys. A15, 92 (2000); M. Anselmino, e-Print Archive: hep-ph/0201150.

[2] X.-N. Wang, Phys. Rev. C61, 064910 (2000); C.-Y. Wong and H. Wang, Phys. Rev. C58, 376 (1998); Y. Zhang, G. Fai, G. Papp, G. Barnaföldi and P. Lévai, Phys. Rev. C65, 034903 (2002);

[3] J.C. Collins, Nucl. Phys. B396, 16 (1993).

[4] D. Sivers, Phys. Rev. D41, 83 (1990); Phys. Rev. D43, 261 (1991).

[5] D. Boer, Phys. Rev. D60, 014012 (1999).

[6] M. Anselmino, D. Boer, U. D'Alesio and F. Murgia, Phys. Rev. D63, 054029 (2001); Phys. Rev. D65, 114014 (2002).

[7] M. Anselmino, V. Barone, A. Drago and F. Murgia, e-Print Archive: hep$\mathrm{ph} / 0209073$.

[8] S.J. Brodsky, D.S. Hwang and I. Schmidt, Phys. Lett. B530, 99 (2002); Nucl. Phys. B642, 344 (2002); J.C. Collins, Phys. Lett. B536, 43 (2002); X. Ji and F. Yuan, Phys. Lett. B543 66, (2002).

[9] M. Gluck, E. Reya and A. Vogt, Z. Phys. C67, 433 (1995).

[10] U. D'Alesio and F. Murgia, work in preparation.

[11] A.S. Ito et al., Phys. Rev. D23, 604 (1981).

[12] S. Kretzer, Phys. Rev. D62, 054001 (2000).

[13] B.A. Kniehl, G. Kramer and B. Pötter, Nucl. Phys. B582, 514 (2000).

[14] G. Donaldson et al., Phys. Lett. B73, 375 (1978).

[15] M. Anselmino, M. Boglione and F. Murgia, Phys. Lett. B362, 164 (1995); M. Anselmino and F. Murgia, Phys. Lett. B442, 470 (1998).

[16] D.L. Adams et al. (E704 Collab.), Phys. Lett. B261, 197 (1991); Phys. Lett. B264, 462 (1991).

[17] M. Anselmino, U. D'Alesio and F. Murgia, e-Print Archive: hep-ph/0210371.

[18] G. Rakness, in proceedings of the 15th International Spin Physics Symposium (SPIN 2002), Long Island, New York, 9-14 Sep. 2002; L.C. Bland, ibidem. 